\title{
Driss Chraibi coordonné par Jeanne Fouet
}

\section{Paola Martini}

\section{(2) OpenEdition}

\section{Journals}

\section{Edizione digitale}

URL: http://journals.openedition.org/studifrancesi/33873

DOI: 10.4000/studifrancesi.33873

ISSN: 2421-5856

\section{Editore}

Rosenberg \& Sellier

\section{Edizione cartacea}

Data di pubblicazione: 1 décembre 2005

Paginazione: 689-691

ISSN: 0039-2944

\section{Notizia bibliografica digitale}

Paola Martini, «Driss Chraibi coordonné par Jeanne Fouet», Studi Francesi [Online], 147 (XLX | III)| 2005,

online dal 30 novembre 2015, consultato il 19 avril 2021. URL: http://journals.openedition.org/

studifrancesi/33873 ; DOI: https://doi.org/10.4000/studifrancesi.33873

\section{Questo documento è stato generato automaticamente il 19 avril 2021.}

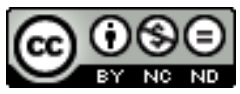

Studi Francesi è distribuita con Licenza Creative Commons Attribuzione - Non commerciale - Non opere derivate 4.0 Internazionale. 


\title{
Driss Chraibi coordonné par Jeanne Fouet
}

\author{
Paola Martini
}

\section{NOTIZIA}

“Expressions maghrébines", dossier Driss Chraibi coordonné par Jeanne FOUET, vol. 3, 2, hiver, 2004, pp. 190.

Il semestrale di cultura maghrebina "Expressions maghrébines" dedica l'ultimo volume dell'annata 2004 a Driss Chraibi, uno degli autori contemporanei più prolifici e poliedrici del Marocco, considerato il padre della letteratura nord africana. Jeanne Fouet, coordinatrice di questo numero, sottolinea nella prefazione l'engagement dello scrittore marocchino, il cui rifiuto nei confronti di ogni pregiudizio e di ogni autoritarismo si è manifestato sempre con forza e humour dirompente.

2 Voies d'accès, la prima sezione della rivista, si apre con un articolo di Hervé ANDERSON тснUмкAM dal titolo Driss Chraibi ou l'éloge de la dissidence: Le passé simple, Les Boucs, Succession ouverte (pp.7-15). Tchumkam prende in considerazione le opere giovanili dello scrittore, tracciando una panoramica delle tematiche ricorrenti e predilette di Chraibi, quali la denuncia delle sclerosi familiari, religiose e sociali, del razzismo e della condizione degli immigrati. La contestazione crea una scrittura che diventa dissenso non solo nei confronti della società coloniale, ma anche dell'opulenta borghesia marocchina. A proposito di Passé simple lo studioso parla di una vera e propria "écriture du refus" (p. 10), laddove attraverso la resistenza e la trasgressione all'idolatria da parte del protagonista nei confronti del padre si delinea la volontà di demistificazione della società marocchina. Della Succession ouverte viene invece messa in risalto la figura della donna, condannata a vivere la sua condizione di emarginata come una fatalità. Nei Boucs la prospettiva è incentrata sugli immigrati e il loro problematico rapporto con la madrepatria da un lato e con il paese d'accoglienza dall'altro, poiché "les immigrés maghrébins sont à cheval entre une mère qui les rejette et les castre: le pays natal, et 
un père qui les marginalise au point de nier leur humanité" (p. 13). Michel LEGRAs ha lavorato sulla ricezione di Boucs (pp. 17-24), romanzo pubblicato nel 1955 che tratta con partecipata emozione e cruda drammaticità del tema dell'immigrazione. L'approccio all'opera da parte della critica - osserva Legras - è divenuto più sereno con gli anni, quando è stato possibile comprendere con maggiore maturità e consapevolezza la portata sociale, politica e ideologica dei suoi contenuti. Le traduzioni, le riedizioni e le ristampe dell'opera che si sono moltiplicate dagli anni sessanta ad oggi costituiscono la testimonianza di un reale successo di pubblico e di critica e ne confermano - per citare le parole dell'editore Denoël - la "poignante actualité" (p. 22). Segue Le Marchand de babouches (pp. 25-27) del romanziere Fouad LAROUI, una breve novella, contenuta nella raccolta intitolata Maboul (Julliard, 2001) in cui viene focalizzata la distanza che separa la società marocchina dall'epoca di Passé simple a quella di oggi. In Coup de cœur en liberté: Interview avec Driss Chraibi (pp. 29-33), Leonor MERINo dà la parola all'autore marocchino attraverso un'intervista in cui egli rievoca gli scrittori che più ha amato $\mathrm{e}$ che l'hanno maggiormente ispirato come Márquez, Borges, Llosa e Eco. L'incontro ha fornito anche l'occasione per parlare di Passé simple e della volontà dello scrittore di dar voce a coloro che voce non hanno, agli emarginati, poiché i suoi libri sono dei romanzi di impegno socio-politico, "un cri d'alarme, une rivolte pour l'accession immédiate à la vie immédiate" (p. 31).

3 La seconda sezione intitolata Grands axes si apre con un contributo di Nadra LAJRI, Regards sur soi (pp. 37-46), in cui romanzo e autobiografia vengono messi a confronto per riflettere sul trattamento letterario del passato, visto ora come tempo idealizzato, ora come momento fortemente criticato. Scrivere il passato non è solo scrivere di sé, ma è anche un impegno letterario e politico che coinvolge la collettività. Lajri si sofferma su alcuni personaggi ricorrenti nei romanzi di Chraïbi, come la madre, figura centrale che emerge per la sua semplicità e per il suo incondizionato amore verso i figli, e il padre, "secrètement admiré et ouvertement rejeté" (p. 37). L'immagine del padre è quella di un personaggio autoritario, intransigente, che simboleggia il potere assoluto, le tradizioni ancestrali, la società marocchina arcaica e conservatrice. Solo più tardi, con $V u$, lu, entendu (1998) il padre non sarà più il simbolo dell'autorità assoluta, ma verrà descritto come un uomo affabile e un pedagogo amabile e aperto. L'evocazione costante dei personaggi del padre e della madre - spiega la studiosa - dimostra la volontà di Chraïbi di esplorare il passato in modo da poter capire e dare un senso al presente. Thomas DEMULDER è l'autore del successivo articolo intitolato L'Homme nouveau à l'ombre de l'olivier (pp.47-60). Il contributo si propone di affrontare uno studio comparato di Une enquête au pays (1981) di Chraïbi e l'opera pittorica di Cherkaoui. I due intellettuali ed artisti marocchini sono impegnati nel recupero, l'uno attraverso la scrittura e l'altro con la pittura, della memoria e delle radici culturali del proprio paese per "dévoiler un sentiment pluriel, multicivilisationnel" (p.48) che accomuna le società del bacino del Mediterraneo. Il sodalizio tra Chraïbi et Cherkaoui è segnato da una ricerca-indagine che li porterà alla scoperta di un entre-deux in grado di rivelare un sentimento di appartenenza "moins tribal, légitime et en parfaite armonie avec la sensibilité de l'homme nouveau" (p.51). Lo scrittore marocchino userà il pretesto dell'indagine poliziesca per passare in rassegna gli indizi di uno spirito mediterraneo: l'ospitalità, le feste, la cucina. Lo stesso itinerario di ricerca identitaria verrà percorso sulle tele da Charkaoui attraverso lo studio della calligrafia e di alcuni simboli ricorrenti, ad esempio, nei tatuaggi e nelle tappezzerie. 
4 La ricerca di un immaginario delle origini è oggetto del successivo articolo De la mère $d u$ printemps (Oum-er-Bia) de Driss Chraibi aux racines méditerranéennes des mythes de la Terre (pp. 63-78), a cura di Bernadette REY-MIMOso-RUIz. La mère du printemps, che si presenta come un inno alla vita e un'ode alla terra perduta, ripercorre l'esodo della tribù degli Aït Yafelman cacciati dalle loro terre dalla spinta conquistatrice islamica. Partendo dal dato storico, Chraïbi sviluppa l'epopea del popolo berbero, assunto come modello di libertà. La studiosa approfondisce il mito originario della Terra-Madre in correlazione con la trasmissione della memoria ad opera delle donne. Ecco allora sfilare figure come Dada, "matrone, cuisinière et sage-femme" (p. 66), conoscitrice di misteriose alchimie e detentrice di un potere primordiale. Il romanzo è al contempo "restitution et création d'un mythe" (p. 73) in cui il métissage di influenze diverse conduce a riscoprire una purezza arcaica comune in un paese così spesso colonizzato. La mère du printemps è oggetto di studio anche di Itsieki PUTU BASEY nel suo articolo L'Espace-temps de la mémoire (pp. 79-95) in cui si sottolinea come l'esercizio della memoria sia l'elemento costitutivo della struttura del testo e crei una vera e propria "poétique du souvenir" (p. 80). L'elaborazione dell'unità dinamica di "espace-temps" da parte di Chraïbi - commenta Putu Basey - crea delle prospettive che spezzando la linearità del discorso danno vita ad un caleidoscopio di immagini suggestive e vivide. La narrazione ripercorre le tappe storiche e mitiche degli antichi popoli che abitavano il Marocco e porta i personaggi a cantare il loro amore per la Madre-Terra. La congiunzione spazio-tempo fonde e confonde realtà e sogno abolendo le opposizioni che separano passato, presente e futuro.

5 L'invito a far tesoro della memoria, a non dimenticare, è al centro della riflessione di Aïcha NEMMAou in Driss Chraibi ou la voix comme identité sans frontières (pp. 97-104). La voce di Chraïbi - osserva l'autrice dell'articolo - con i suoi canti e i suoi silenzi, diventa "modulation identitatire" (p. 102) ed evidenzia una dialettica in continua variazione ed evoluzione. Ecco allora che l'esercizio della memoria permette la riconciliazione col passato e la speranza nell'avvenire.

6 Estelle MALESKI, con L'inspecteur Ali m'a tué...(pp. 107-121), apre la terza ed ultima sezione intitolata Chemins de traverse. Il contributo offre l'occasione di riflettere sulla scelta da parte di Chraïbi di scrivere romanzi polizieschi, genere notoriamente di tradizione popolare e d'ispirazione occidentale. Apprenderemo dunque che l'universalità e la semplicità della forma poliziesca - ampiamente diffusa in tutto il mondo - permette di trattare le questioni culturali più complesse con una leggerezza tale da renderne la lettura facilmente fruibile per la società odierna in continua evoluzione. Il genere poliziesco, ripreso anche da alcuni autori delle Antille francofone come Chamoiseau e Confiant, abbandona con Chraïbi gli stilemi più classici della forma tradizionale per dare risalto alla ricerca identitaria e alla peculiarità degli ambienti postcoloniali. Il romanzo poliziesco diventa così terreno di esperienze e riflessioni di tipo metaletterario e, come spiega Maleski, "c'est finalement le roman qui parait se costruire au fil de l'enquête, porté à bout de bras par la puissance de l'acte narratif" (p.117). In Le Couple incestueux dans l'oeuvre de Driss Chraibi (pp.123-134) Anne Marie GANS-GUINOUNE riflette, alla luce delle teorie etno-psicologiche, sulla modalità con cui leggere una storia d'amore tra padre e figlia quando questa viene raccontata da uno scrittore maghrebino. Il contributo ci spiega che l'intento dello scrittore marocchino non è quello di fare un'apologia degli amori proibiti, ma di trasportarci verso un "monde fantasmatique" (p.123) in cui è possibile dar vita a quel fantasma che 
ossessiona i giovani fanciulli innamorati della propria madre. La sostituzione, all'interno del contesto amoroso, della coppia madre/figlia con quella padre/figlia commenta Gans-Guinoune - consente di alleggerire l'opera da tensioni e carica emotiva. La dinamica edipica è riscontrabile fin dal Passé simple, il cui testo può essere letto come un lungo grido d'amore del protagonista Driss rivolto alla madre, ma anche al padre nei confronti del quale il sentimento si tinge di un inevitabile senso di colpa.

7 Jeanne FOUET, nell'articolo che segue intitolato Les Contes pour enfants de Driss Chraibi (pp. 135-139), ci propone la rilettura di una parte meno conosciuta della produzione di Chraïbi: i racconti per l'infanzia. Il fortunato ciclo dell' Âne K'hal ha potuto vantare l'inserimento nel corpus narrativo di un buon numero di illustrazioni, volte a realizzare il progetto pedagogico dello scrittore marocchino: "expliquer à un petit Français plusieurs mots du vocabulaire de la vie quotidienne au Maroc" (p. 138). Il simpatico animale - osserva Fouet - costituisce anche un'importante fonte d'ispirazione per la creazione del personaggio dell'Ispettore Ali, protagonista dei romanzi polizieschi parodici di Chraïbi. Il dossier si conclude con il contributo di Guy DUGAS, Une Mémoire en train de se constituer: Interview avec Driss Chraibi (pp. 141-177). Nell'intervista, concessa a Dugas poco dopo la pubblicazione delle sue memorie Vu, lu, Entendu (1998), Chraïbi coglie l'occasione per spiegare che non bisogna etichettare rigidamente la sua opera come autobiografica, poiché il suo intento è stato principalmente quello di parlare degli altri, della gente che ha conosciuto in Marocco e altrove, nelle diverse età della sua vita. Più volte lo scrittore ritorna sul concetto di memoria e passato e non perderà occasione per ricordare che "le présent demande à être éclairé par le passé". Per comprendere il mondo attuale - insiste lo scrittore - bisogna assolutamente interrogare il passato. 